\title{
Pourquoi les femmes informent-elles plus souvent leurs partenaires sexuels d'une infection sexuellement transmissible ? Rôle des médecins
}

\author{
Josiane WARSZAWSKI ${ }^{1}$, Elisabeth COUTURIER ${ }^{2}$, Emile RIBERT ${ }^{3}$, Laurence MEYER ${ }^{4}$
}

\begin{abstract}
Résumé
Des travaux menés à partir d'enquêtes nationales ont montré qu'à l'occasion d'une maladie sexuellement transmissible, les patients préviennent en général leur partenaire principal, mais les hommes sont moins nombreux que les femmes à le faire. En revanche, quel que soit leur sexe, ils préviennent plus rarement d'autres partenaires sexuels.

Compte tenu du rôle des médecins dans la prise en charge de ces infections, les pratiques de 215 d'entre eux exerçant en région parisienne ont été analysées, à l'occasion d'un diagnostic récent de maladie sexuellement transmissible ou de VIH. La majorité d'entre eux incitent leurs patients à informer leur partenaire principal, mais se préoccupent moins nettement des partenaires occasionnels ou plus anciens. Les gynécologues sont les plus actifs à cet égard, à l'opposé des cliniciens exerçant dans des dispensaires antivénériens ou des structures hospitalières.

II apparait ainsi que les hommes consultent dans des structures où on les incite moins activement à prévenir leurs partenaires. La conséquence peut en être un retard au diagnostic pour les femmes porteuses d'une infection asymptomatique, avec un impact potentiel sur leur santé en matière de reproduction.
\end{abstract}

Mots clés : infection sexuellement transmissible, VIH, santé publique, épidémiologie.

\section{Prévention des infections sexuellement transmissibles et du VIH : intérêts et limites de l'information des partenaires sexuels}

L'absence de signes d'alerte est le principal obstacle au dépistage précoce des infections sexuellement transmissibles et du VIH, particulièrement chez les femmes qui, en outre, supportent l'essentiel de leurs complications. L'infection à Chlamydia trachomatis, la plus fréquente de ces infection, passe le plus souvent inaperçue, et l'extension du germe vers les voies génitales hautes survient dans 10 à $20 \%$ des cas chez les femmes. Deux types de situations méritent d'être distingués. Certaines

\footnotetext{
Unité Inserm 822/Ined, Université Paris 11, AP-HP, Hôpital de Bicêtre, le Kremlin Bicêtre. Institut national de veille sanitaire, St-Maurice.

CETSAH, CNRS

Unité Inserm 822/Ined, Université Paris 11, AP-HP, Hôpital de Bicêtre, le Kremlin Bicêtre.
}

infections (chlamydiose, gonococcie, syphilis) peuvent être guéries rapidement par une antibiothérapie simple et bien tolérée : administrée suffisamment tôt, celle-ci empêche l'apparition de séquelles et abrège la période pendant laquelle le sujet infecté risque de contaminer ses partenaires sexuels. D'autres infections ne bénéficient pas de traitement curatif et évoluent généralement sur un mode chronique, mais leur dépistage précoce garde néanmoins tout son intérêt pour : détecter un éventuel cancer du col en cas de présence de papilloma virus (HPV), à un stade où il est possible d'en assurer la guérison ; introduire pendant la grossesse une stratégie prophylactique efficace pour éviter la transmission verticale du VIH ou de l'herpès génital; inciter les personnes porteuses du germe à protéger leurs partenaires sexuels par l'usage de préservatifs.

L'information des différents partenaires sexuels estl'un des volets habituels des programmes de prévention des infections sexuellement transmissibles, à côté de stratégies de dépistage systématique dans des populations asymptomatiques. Elle permet d'atteindre des personnes ayant un risque élevé d'être infectées, sans le savoir, par un germe. Le but est triple : limiter le risque de recontamination du patient, limiter le risque de complications chez les partenaires dépistés, limiter la propagation de l'agent infectieux dans la population. Ce dernier point est fondamental en termes de santé publique, la lutte contre les infections sexuellement transmissibles, actuellement en recrudescence, ne pouvant reposer exclusivement sur des actions visant à favoriser l'adoption de comportements sexuels à moindre risque.

Les législations et recommandations nationales concernant les différents aspects du contrôle des infections sexuellement transmissibles sont variables d'un pays à l'autre [1]. Dans les pays anglo-saxons et nordiques, un effort important a été consacré à la promotion de stratégies de dépistage systématique et de notification des partenaires [2]. Des professionnels de santé sont formés spécialement pour ces activités, et la notification des partenaires y est fortement recommandée, voire obligatoire [3,4]. Certaines approches supposent que le patient fournisse, au cours d'un entretien avec un professionnel de santé, toutes les informations utiles pour identifier et retrouver ses différents partenaires sexuels sur une période variable selon le germe concerné. Ce 
professionnel incite le patient à les avertir lui-même, mais engage ensuite les démarches pour avertir à sa place les partenaires qui ne se sont pas manifestés, dans un certain délai, auprès d'un service de soins (approche dite " provider ou conditional refer$\mathrm{ral}$ »). Des approches alternatives existent, qui ne nécessitent pas la divulgation de l'identité des partenaires sexuels. Le personnel de santé est là pour déterminer, avec le patient, les partenaires à informer, l'encourager à le faire en lui donnant des conseils sur l'information à transmettre et sur les procédés à utiliser (patient referral, patient index). II peut également remettre au patient des documents destinés aux partenaires (lettre, formulaire, carte ou brochure, adresses de services de soins à contacter etc) [5]. Dans quelques pays, le patient peut recevoir des traitements ou une ordonnance à remettre directement à ses partenaires, sans dépistage [6]. Cette pratique, réservée en général aux infections à Chlamydia trachomatis ou à gonocoque est rare en Europe. Un essai randomisé américain récent a montré l'efficacité de la remise d'un traitement monodose aux partenaires sexuels par l'intermédiaire du patient ou d'un personnel qualifié, pour diminuer le risque de recontamination par Chlamydia trachomatis et Neisseria gonorrhoeae [7]. L'évaluation des procédures de notification est difficile et les résultats ne sont pas toujours concluants $[8,9,10]$. La première approche (provider et conditional referra) s'avère nettement plus coûteuse et sa supériorité sur la seconde (patient referral) n'est pas toujours évidente [2]. Les efforts passés à conseiller le patient index, ainsi que la formation du personnel qui s'en charge semblent être des facteurs d'efficacité du processus [11,12].

Dans les pays où des services de conseils et d'information des partenaires par un personnel formé existent, ces services dépendent généralement de dispensaires antivénériens [13] et sont peu accessibles aux patients pris en charge par des praticiens géographiquement dispersés [14]. Cela constitue un obstacle à leur développement dans les pays où la plupart des infections sexuellement transmissibles sont diagnostiquées en dehors de ces structures, ce qui est le cas de la France.

\section{La situation française}

En France, il n'existe ni recommandation nationale, ni professionnels de santé spécialisés dans l'information des partenaires. L'idée que des méthodes standardisées et évaluées pour aider les patients à notifier leurs partenaires, avec éventuellement une formation spécifique ou un personnel dédié, est peu connue ou considérée avec méfiance. En outre, l'application de ces stratégies est compliquée par la dispersion et la diversité des lieux de diagnostics des infections sexuellement transmissibles. Les structures publiques prennent en charge moins de $20 \%$ de l'ensemble de ces infections et sont hétérogènes ${ }^{5}$. Parmi elles, les

\footnotetext{
5 Le diagnostic peut être fait à l'hôpital, en consultation spécialisée de gynécologie, d'urologie, de dermatologie, mais aussi en consultation de médecine générale, dans le cadre d'une hospitalisation pour une complication, dans des dispensaires antivénériens ou des centres de planification familiale.
}

dispensaires antivénériens et les centres de planification familiale sont les seules structures dont la mission est de dépister et de traiter gratuitement ces infections. La majorité des infections sexuellement transmissibles est donc vue dans le secteur privé (généralistes et gynécologues libéraux), mais chaque praticien a individuellement peu d'occasions d'en voir [15]. Les frais relèvent du régime de l'assurance maladie auquel est assujetti le patient.

Plusieurs enquêtes nationales sur les comportements sexuels ou la santé (ACSF 92, ACSJ94, KABP, Baromètre santé) ont permis d'explorer les pratiques de personnes ayant rapporté un antécédent d'infection sexuellement transmissible $[16,17]$. Ce diagnostic a un impact plus marqué sur les comportements ultérieurs des femmes que des hommes, que ce soit en termes d'information de leurs partenaires sexuels, de comportements sexuels ultérieurs ou de craintes de nouvelles infections [18]. La majorité des sujets déclarait avoir prévenu leur partenaire principal à l'occasion d'une infection sexuellement transmissible, mais les hommes étaient plus nombreux que les femmes à ne pas l'avoir fait, avec un écart important chez les adolescents. Dans chacune des enquêtes, l'association avec le sexe était statistiquement significative et indépendante du type d'infection, de l'âge, du niveau d'études et du nombre de partenaires. Cette différence d'attitude se traduit d'ailleurs en miroir dans les circonstances de diagnostic, les hommes ayant été trois fois plus souvent que les femmes avertis par leur partenaire. L'information d'autres types de partenaires sexuels était plus rare et sans relation avec le sexe du patient.

Le rôle possible de l'attitude des médecins et de la structure médicale où est effectué le diagnostic est suggéré indirectement par les déclarations des sujets. Les femmes rapportaient plus souvent que les hommes avoir parlé avec leur médecin des risques de cette infection pour leur santé, de leur sexualité et de la manière d'informer leurs partenaires sexuels. Or ces échanges ont eu lieu essentiellement avec un gynécologue libéral pour les femmes, un généraliste et plus rarement une structure publique pour les hommes. Les patients ont d'ailleurs moins souvent informé leurs partenaires lorsque le diagnostic a été réalisé dans une structure publique que chez un spécialiste privé ou un généraliste [17].

Ces résultats nous ont conduits à explorer auprès des médecins les modalités de prise en charge et d'information des patients et de leurs partenaires à l'occasion d'un diagnostic d'infection sexuellement transmissible, et à analyser leurs pratiques et attitudes selon leur structure d'exercice et le type d'infection en cause [19]. Nous nous sommes restreints à celles dont la morbidité est sévère : trois infections bactériennes classiques et facilement curables (chlamydia, gonocoque, syphilis) et l'infection à VIH. L'analyse présentée dans la partie suivante porte sur l'information des partenaires sexuels: les médecins évoquentils cette question et à propos de quels partenaires (partenaire principal ou régulier, partenaires occasionnels, partenaires plus 
anciens, partenaires dans le cadre d'un rapport de prostitution...), comment envisagent-ils de faire prévenir le partenaire (directement, par téléphone, par l'intermédiaire d'une tierce personne...), remettent-ils un document pour ce(s) partenaire(s) (brochure, ordonnance de traitement, ordonnance de dépistage, lettre du médecin...)?

\section{Attitudes des médecins vis-à-vis de l'information des partenaires sexuels de leurs patients}

\section{Méthodologie de l'enquête auprès des médecins en région parisienne}

Le volet principal de l'étude a consisté à sélectionner des médecins par l'intermédiaire de laboratoires de biologie médicale de la région parisienne ${ }^{6}$. Parmi eux, 66 ont accepté de recenser entre décembre 2001 et mars 2002 tous les nouveaux diagnostics de Neisseria gonorrhoeae, Chlamydia trachomatis, syphilis ou $\mathrm{VIH}^{7}$. Le laboratoire informait le médecin prescripteur que nous souhaitions l'interroger sur la prise en charge des infections sexuellement transmissibles et nous communiquait ses coordonnées en l'absence de refus (il n'y en a eu aucun à ce stade). Un enquêteur prenait contact par téléphone avec ce médecin pour lui proposer un rendez-vous. Afin d'éviter que le médecin ne prépare ses réponses, l'enquêteur n'indiquait pas que le questionnaire porterait sur un patient en particulier. Le questionnaire était administré sur leur lieu d'exercice et explorait la prise en charge du patient index à l'origine de l'inclusion du médecin dans l'étude. Les médecins qui ne se souvenaient pas de ce patient étaient invités à parler d'un autre patient récemment diagnostiqué, si possible pour le même germe. Chaque médecin n'était interrogé qu'une seule fois.

Compte tenu de la rareté attendue des diagnostics effectués dans les dispensaires antivénériens et les centres de planification familiale, et de leur mission spécifique dans ce domaine, un volet complémentaire a été réalisé entre avril et juin 2002 auprès de médecins tirés au sort dans les dispensaires antivénériens de Paris et dans un échantillon de centres de planification familiale de Paris ou d'une commune limitrophe. Le questionnaire portait alors sur la prise en charge du dernier patient vu pour l'une des infections ciblées par l'étude. La participation des centres et des dispensaires n'a pu être sollicitée qu'à la fin du volet principal, afin de permettre en priorité l'inclusion des médecins par l'intermédiaire des laboratoires.

Cette enquête a été conçue de manière à éviter deux types d'écueils : sous-estimer la fréquence avec laquelle l'information

\footnotetext{
6 Membres d'un réseau sentinelle de surveillance de certaines maladies sexuellement transmissibles ou du VIH (RENACHLA, Renago, ex Renavi) dans les départements 75, 92, 93, 94, soit au total 91 laboratoires éligibles.

7 Définis par: recherche génito-urinaire, anale ou pharyngée, positive de gonocoque ou de chlamydia, VDRL et/ou TPHA positif et/ou un autre examen sérologique syphilitique, Western blot pour le VIH positif ou évocateur d'une séroconversion.
}

du partenaire n'était pas du tout abordée au cours de la consultation ; ne pas distinguer une incitation générale, sans doute peu efficace (« il faut prévenir vos partenaires sexuels »), d'une attitude active consistant à identifier chaque partenaire à informer et la manière de le faire. Une enquête pilote qualitative avait été menée au préalable par une sociologue auprès de vingt médecins sélectionnés dans les mêmes conditions que dans le volet principal. Elle a contribué à affiner les hypothèses, à construire le questionnaire standardisé et à éclairer a posteriori une partie de ces résultats.

Les principes du questionnaire ont été les suivants: $1^{\circ}$ ) interroger le médecin en face-à-face au moyen d'un questionnaire standardisé d'une trentaine de minutes comportant des questions fermées et ouvertes ; $2^{\circ}$ ) faire décrire la prise en charge d'un patient chez qui le médecin avait fait récemment un diagnostic d'infection sexuellement transmissible, afin d'éviter de recueillir l'idée plus ou moins idéalisée qu'il se fait de sa pratique, ou de ce qu'elle devrait être ; $3^{\circ}$ ) organiser cette description dans l'ordre chronologique, en commençant par le motif de la consultation initiale, les choix thérapeutiques, l'orientation éventuelle vers une autre structure médicale, cela afin que la question de la prise en charge des partenaires n'apparaisse pas comme l'élément central $; 4^{\circ}$ ) terminer par un questionnaire général sur les attitudes et opinions sur la prévention et le counselling.

Nous avons d'abord décrit la distribution des différentes stratégies de prise en charge du partenaire principal, puis des autres partenaires sexuels, selon le type de structure médicale. Puis nous avons mené une analyse univariée (tests de chi2 ou de Fisher) et multivariée (régression logistique) pour identifier les facteurs associés à deux pratiques pouvant refléter un engagement actif et concret : remettre un document au patient à l'intention de son partenaire principal ; évoquer l'information d'autres partenaires en ciblant certains d'entre eux en particulier. L'analyse statistique a été menée avec le logiciel Stata.

\section{Participation des médecins}

Les laboratoires ont identifié 227 médecins prescripteurs différents, ayant diagnostiqué les infections suivantes : 81 infections à VIH, 75 Chlamydia trachomatis, 49 syphilis et 22 Neisseria gonorrhoeae. Parmi eux, 185 (82\%) ont accepté l'entretien. Au total, 129 médecins ont pu décrire la prise en charge du patient index et 25 celle d'un patient de remplacement vu récemment pour la même infection. Les 31 autres, qui ne se rappelaient plus ni du patient index, ni d'un autre patient éligible, ont répondu à des questions générales sur leurs attitudes et n'ont pas été inclus dans cette analyse. Le volet complémentaire a permis de tirer au sort 60 médecins exerçant dans les dispensaires antivénériens et les centres de planification familiale, dont 38 étaient disponibles pour recevoir l'enquêteur et 33 ont pu répondre à propos d'un patient précis. II s'agissait d'un diagnostic de VIH dans $80 \%$ des cas décrits par un médecin de dispensaire anti- 
Tableau 1

Prise en charge des partenaires sexuels des patients par les médecins ayant diagnostiqué l'infection sexuellement transmissible Enquête auprès des médecins 2002

\begin{tabular}{|c|c|c|c|c|c|c|c|c|}
\hline & \multicolumn{2}{|c|}{$\begin{array}{l}\text { Total } \\
n=187\end{array}$} & \multicolumn{2}{|c|}{$\begin{array}{c}\text { Privé sauf } \\
\text { gynécologue } \\
n=60\end{array}$} & \multicolumn{2}{|c|}{$\begin{array}{c}\text { Gynécologue } \\
\text { et CPF* } \\
n=30\end{array}$} & \multicolumn{2}{|c|}{$\begin{array}{c}D A V^{\star \star} \\
\text { et hôpital } \\
n=97\end{array}$} \\
\hline & $\%$ & (n) & $\%$ & (n) & $\%$ & (n) & $\%$ & (n) \\
\hline Prise en charge du partenaire principal & $n=122$ & & $\mathrm{n}=37$ & & $\mathrm{n}=27$ & & $\mathrm{n}=58$ & \\
\hline Patient venu parce que son partenaire principal était infecté & 8,2 & $(10)$ & 8,1 & (3) & 3,7 & (1) & 10,3 & (6) \\
\hline Partenaire principal vu par le médecin en consultation & 13,9 & (17) & 16,2 & (6) & 22,2 & (6) & 8,6 & (5) \\
\hline Remise au patient d'un document pour le partenaire & 29,5 & (36) & 18,9 & (7) & 66,7 & $(18)$ & 19,0 & (11) \\
\hline Information du partenaire discutée avec le patient & 34,5 & (42) & 48,7 & (18) & 7,4 & (2) & 38,0 & (22) \\
\hline Pas de discussion sur l'information du partenaire & 13,9 & (17) & 8,1 & (3) & 0,0 & (0) & 24,1 & (14) \\
\hline Prise en charge d'autres types de partenaires & $n=105$ & & $n=47$ & & $n=5$ & & $n=53$ & \\
\hline Information ciblée sur certains partenaires ou remise de document ${ }^{*}$ & 24,8 & (26) & 29,8 & (14) & 60,0 & (3) & 17,0 & (9) \\
\hline Recommandations générales sur l'information des partenaires & 47,6 & $(50)$ & 48,9 & (23) & 40,0 & (2) & 47,2 & (25) \\
\hline N'ont pas discuté de l'information de ces partenaires & 27,6 & (29) & 21,3 & (10) & 0,0 & (0) & 35,8 & (19) \\
\hline
\end{tabular}

- Centres de planification familiale.

* Dispensaires antivénériens.

... Après exclusion des patients qui n'ont pas eu d'autres partenaires dans les 12 derniers mois.

*** Une ordonnance de dépistage pour une syphilis; deux ordonnances de traitement et une ordonnance de dépistage pour un Chlamydia trachomatis.

vénérien et de Chlamidia trachomatis dans $72 \%$ des cas décrits par un médecin de centres de planification familiale. Au total, la prise en charge de 187 patients a pu être décrite en détail. Le profil de l'échantillon sélectionné par le biais des laboratoires est cohérent avec les différentes sources de surveillance françaises des infections sexuellement transmissibles quant à la répartition des diagnostics, des types de prescripteurs et leur forte corrélation avec le sexe [17, 20, 21].

\section{Attitude des médecins selon le statut du partenaire}

Les réponses des médecins témoignent d'une connaissance limitée du réseau partenarial de leur patient: la majorité des médecins connaissait son orientation sexuelle et savait s'il était en couple ou avait un partenaire principal, mais $23 \%$ ignoraient si le partenaire principal était nouveau ou non, $26 \%$ ignoraient s'il y avait eu plusieurs partenaires sexuels dans l'année, et $8 \%$ ne savaient rien sur les partenaires de leur patient.

La majorité des médecins $(86,1 \%)$ s'est préoccupée de l'information du partenaire principal (tableau 1). Dans $8,2 \%$ des cas, ce partenaire a été la source du dépistage chez le patient; dans $13,9 \%$ des cas, les médecins l'ont vu en même temps que le patient ou après lui. Sinon, près d'un tiers des médecins $(29,5 \%)$ lui ont fait remettre un document par l'intermédiaire du patient. II s'agissait le plus souvent d'une ordonnance de traitement (26/36), surtout en cas d'infections à Chlamydia trachomatis, moins fréquemment d'une ordonnance de dépistage (10/36), surtout pour la syphilis et le gonocoque et, plus rarement encore, d'une lettre ou d'une brochure (8/36). Enfin un tiers des médecins $(34,4 \%)$ s'est contenté de conseils verbaux sans rien remettre, invoquant le plus souvent, comme raison de n'avoir rien remis, que le partenaire était déjà au courant ou allait être averti par le patient, ou que le médecin voulait le voir en consultation.

L'information d'autres partenaires n'a pas été évoquée dans $27,6 \%$ des cas (tableau 1), moins encore lorsque le patient n'avait pas de partenaire principal $(35,4 \%)$. Elle l'a été sous forme d'une recommandation générale dans la moitié des cas $(47,6 \%)$, beaucoup plus rarement en identifiant précisément un certain nombre de partenaires à prévenir $(21,0 \%)$. La remise d'un document pour un autre partenaire que le partenaire principal actuel a été exceptionnelle $(3,8 \%)$.

\section{Attitudes des médecins selon leur spécialité et le type de structure dans laquelle ils exercent}

Les gynécologues libéraux et les médecins de centres de planification familiale ont diagnostiqué essentiellement des infections féminines à Chlamydia trachomatis (87 \%) ; les médecins généralistes et spécialistes (hors gynécologues) exerçant dans un cabinet libéral ou une clinique privée ont diagnostiqué principalement des infections sexuellement transmissibles masculines (90\%), le plus souvent une syphilis ( $28 \%$ ) ; les structures publiques (hôpitaux, dispensaires antivénériens) ont reçu un tiers de femmes et une majorité d'infections à VIH $(56 \%)$. Cette répartition est conforme aux données de surveillance épidémiologique.

Les gynécologues libéraux et les médecins de centres de planification familiale savaient tous si leur patiente avait ou non un partenaire principal au moment de la consultation et affirmaient que c'était le cas de $90 \%$ d'entre elles. Ces médecins se sont toujours préoccupés de l'information du partenaire principal, l'ont vu en consultation dans $22,2 \%$ des cas ou le plus souvent 
Tableau 2

Analyses univariées et multivariées de la prise en charge du partenaire principal et d'autres types de partenaires, selon le sexe, l'âge, le diagnostic et la structure médicale - Enquête auprès des médecins (2002)

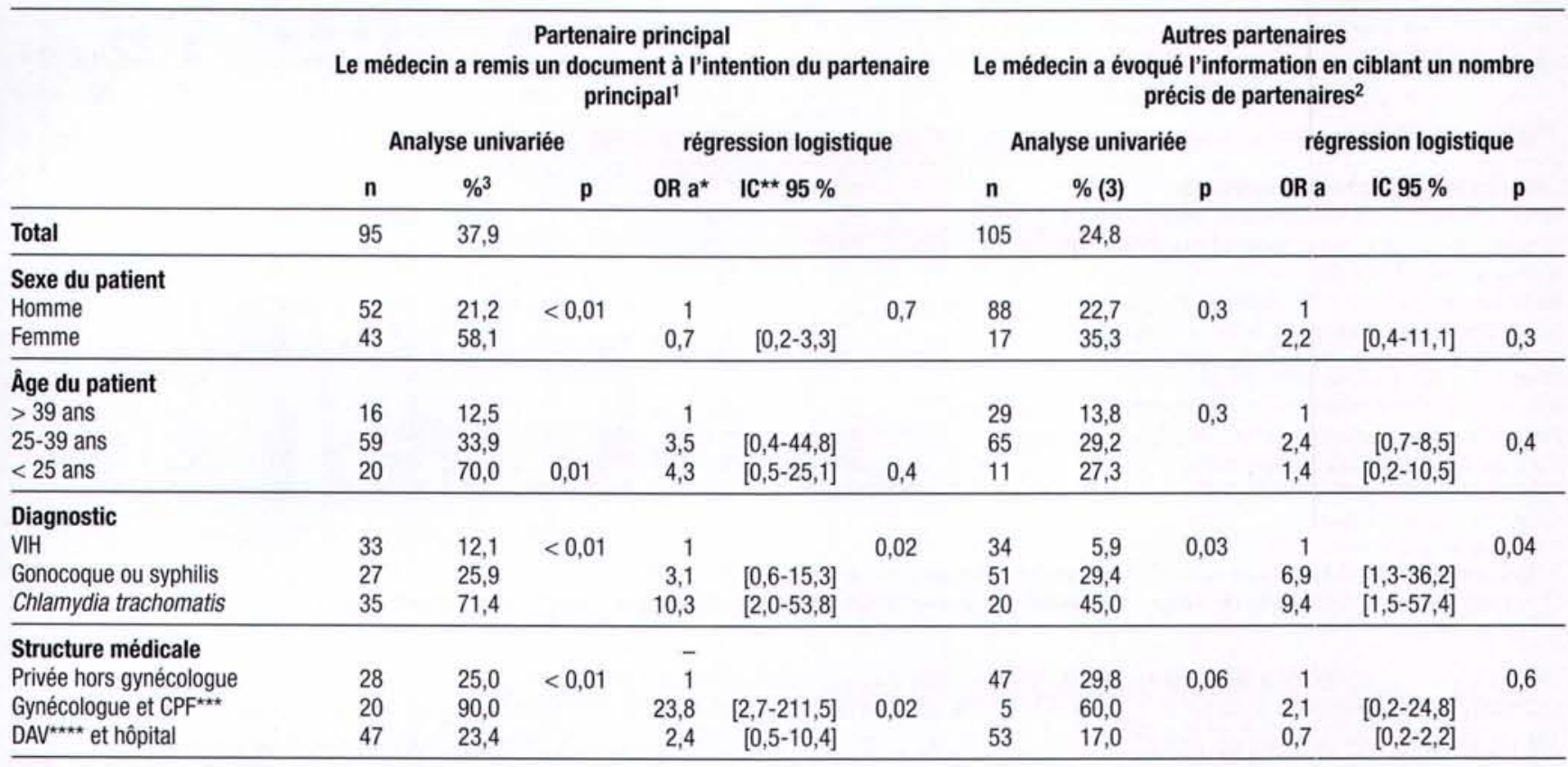

' Les patients dont le partenaire principal avait déjà été dépisté ou a été vu en consultation par le médecin sont exclus.

${ }^{2}$ Les patients qui n'ont pas eu d'autre partenaire dans l'année qu'un partenaire principal sont exclus.

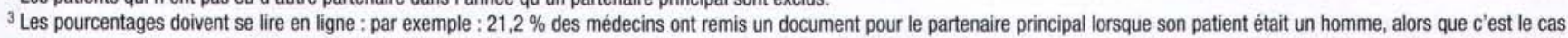
de $58,1 \%$ des médecins dont le patient était une femme.

* odds ratio ajusté par régression logistique ;

** Intervalle de confiance à $95 \%$ de l'odd ratio :

*** centres de planification familiale ;

**** dispensaire antivénérien.

lui ont fait remettre une ordonnance par l'intermédiaire de la patiente $(66,7 \%)$. Lorsqu'ils ne l'ont pas fait, c'est qu'ils disaient savoir que le partenaire était averti ou allait l'être. Ils ont aussi évoqué explicitement l'information des autres partenaires pour les patientes éventuellement concernées, situation rare d'après leur déclaration.

Les autres cliniciens libéraux connaissaient également assez bien les patients chez qui ils ont diagnostiqué une infection sexuellement transmissible ou une infection à $\mathrm{VIH}$ : deux tiers de leurs patients avaient des partenaires du même sexe, $32 \%$ n'avaient pas de partenaire principal et $62 \%$ avaient eu plusieurs partenaires dans l'année, dont le médecin, dans la plupart des cas, ignorait le nombre. Les médecins ont vu le partenaire principal dans $16,2 \%$ des cas, ont incité oralement le patient à l'avertir dans $48,7 \%$ des cas et lui ont fait remettre une ordonnance dans $18,9 \%$ des cas. L'information des autres partenaires a été évoquée sur un mode général dans la moitié des cas. Ces médecins sont tout de même $8,1 \%$ à ne pas avoir parlé de l'information du partenaire principal et $21,3 \%$ à ne pas avoir évoqué celle d'autres partenaires, les pensant non joignables ou trouvant le sujet difficile à aborder avec le patient.

En revanche, les médecins interrogés en dispensaires antivénériens ou dans des structures hospitalières connaissaient mal le patient dont ils ont rapporté la prise en charge : ils étaient plus nombreux à l'avoir vu pour la première fois, à ne pas l'avoir revu après la consultation initiale, à ne pas connaître sa profession, son niveau de revenu, son orientation sexuelle, et à ne pas savoir s'il avait un partenaire principal et/ou plusieurs partenaires sexuels. Ils ont abordé beaucoup moins souvent que les autres cliniciens la question de l'information des partenaires. Ils ne l'ont pas fait à propos du partenaire principal dans $24,2 \%$ des cas et encore moins à propos d'autres types de partenaires $(35,8 \%)$. C'est dans les dispensaires antivénériens qu'une ordonnance pour un partenaire a été le plus rarement remise.

Les facteurs lié au fait de remettre au patient une ordonnance à l'intention de son partenaire principal sont présentés dans le tableau 2: sexe féminin, jeune âge, diagnostic d'infection bactérienne (surtout une infection à chlamydia), et structure médicale 
où a été effectué le diagnostic (gynécologue privé ou centre de planification familiale). Après ajustement sur ces variables dans une régression logistique, seule la liaison avec le type de germe et de structure médicale persiste, alors que la liaison avec le sexe et l'âge disparaît. Mais la corrélation étroite entre le sexe du patient, le type de germe dépisté et la structure médicale où a été prescrit l'examen microbiologique ne permet pas d'individualiser le rôle propre de chacun de ces facteurs. L'évocation de partenaires précis à informer en dehors du partenaire principal, réalisée par un quart seulement des médecins, présente un profil d'association assez similaire, mais seul le type d'infection reste liée à cette pratique : $5,9 \%$ des médecins l'ont fait pour un patient infecté par le VIH, alors que c'est le cas de $45 \%$ des médecins face à une infection à chlamydia.

\section{Conclusion}

Peu de travaux ont été menés en France sur la prise en charge des infections sexuellement transmissibles par les médecins. La double approche que nous avons adoptée, à la fois quantitative et qualitative, permet de mieux comprendre le rôle des médecins dans les attitudes des patients à l'égard de leurs partenaires, en particulier, les différences entre hommes et femmes.

C'est ainsi que nous avions montré dans plusieurs enquêtes en population générale qu'à l'occasion d'un diagnostic d'infection sexuellement transmissible, les patients informent généralement leur partenaire principal mais beaucoup plus rarement d'autres partenaires sexuels, et qu'ils les informent d'autant moins qu'ils ont été diagnostiqués dans une structure publique. Notre étude auprès des médecins suggère leur influence possible sur ces comportements : la majorité d'entre eux incitent en effet leurs patients à prévenir leur partenaire principal mais sont peu nombreux à s'inquiéter des partenaires occasionnels ou plus anciens et c'est bien dans les dispensaires antivénériens ou dans les hôpitaux qu'ils abordent le moins cette question. Un tiers des médecins affirme d'ailleurs ne pas avoir parlé du tout des partenaires lorsque leur patient n'avait pas de partenaire principal.

II faut noter toutefois que l'on est loin des méthodes de notification des partenaires recommandées en Grande-Bretagne, dans les pays scandinaves ou aux États-Unis. Ces méthodes supposent une première étape consistant à établir avec le patient la liste des différents partenaires à informer en remontant, selon les germes, à 6 ou 12 mois en arrière. Le but est de ne pas oublier des partenaires occasionnels ou d'anciens partenaires susceptibles d'être porteurs de l'infection et d'évaluer avec le patient les moyens pratiques d'avertir de façon adaptée chacun de ces partenaires. Or les médecins interrogés dans notre étude n'ont manifestement pas mené des investigations poussées sur le réseau partenarial de leur patient. Concernant d'autres partenaires que le partenaire principal, la moitié environ des médecins déclare s'être contentée de recommandations générales et un quart seulement affirme avoir ciblé certains partenaires en particulier. Ce dernier pourcentage est vraisemblablement surestimé car 9 des 22 médecins qui déclarent avoir déterminé avec leur patient un nombre précis de partenaires à prévenir ont déclaré, ailleurs dans le questionnaire, ne pas savoir si leur patient avait eu d'autres partenaires dans l'année que le partenaire principal actuel, ni combien il en avait eu. L'information des partenaires sexuels autres que le partenaire principal apparaît ainsi une préoccupation secondaire pour les médecins qui prennent en charge une infection sexuellement transmissible, et plus encore une infection à VIH.

L'enquête qualitative initiale montrait bien que, d'une manière générale, les médecins ont une conception individuelle de la prise en charge : s'ils insistent sur l'information et le traitement des partenaires avec lesquels le patient a encore des relations, c'est pour éviter une recontamination et ils visent alors exclusivement le partenaire principal. En outre, les médecins ne veulent pas paraitre normatifs, car ils pensent cette attitude mal acceptée des patients. Ils se sentent particulièrement impuissants face aux patients qui, tout en étant bien informés, ont des comportements à risque et de multiples partenaires. Les médecins pensent que des conseils concernant des partenaires occasionnels sont inutiles car trop nombreux ou injoignables, que le patient ne souhaite pas recontacter un ancien partenaire, qu'un patient qui ne s'est pas protégé avec un partenaire occasionnel ne va pas se soucier de la santé de celui-ci et faire la démarche de le prévenir.

Nous avions rapporté en population générale une nette différence de comportement selon le sexe, les femmes étant plus nombreuses que les hommes à informer leur partenaire principal à l'issue du diagnostic. Cette différence tient sans doute en grande partie au fait que les femmes sont diagnostiquées par des gynécologues libéraux et des médecins de centres de planification familiale qui les encouragent et les aident à le faire. Ces cliniciens veulent éviter la recontamination de leur patiente et n'hésitent pas à remettre une ordonnance de dépistage ou de traitement pour le partenaire principal. Mais ils dépistent surtout des infections à Chlamydia trachomatis, auxquelles ils sont sensibilisés en raison du risque de séquelles sur la fonction reproductive et qu'ils peuvent facilement rechercher par un prélèvement au cours de l'examen de routine du col de l'utérus. La majorité des diagnostics masculins sont effectués dans des structures où l'information du partenaire se résume au mieux à une recommandation orale, mais il ne s'agit pas des mêmes germes en général : une syphilis chez des patients homosexuels suivis par des généralistes ou une infection à VIH représentant la moitié des diagnostics rapportés par les dispensaires antivénériens et les hôpitaux. Or le moment de la découverte d'une infection à VIH est une période peu opportune pour aborder la question de l'information des partenaires qui ne présente pas de bénéfice direct pour le patient. 
C'est ainsi que la spécialité et les conditions d'exercices des cliniciens, dans la mesure où elles déterminent leurs centres d'intérêt et leurs compétences spécifiques ainsi que le profil sociodémographique de leurs patients, conditionnent l'ensemble du processus de dépistage et de prise en charge, depuis le type de germes recherchés chez des personnes asymptomatiques jusqu'aux conseils de prévention prodigués. L'enquête qualitative a permis de bien observer l'opposition entre structures publiques et médecine de ville. Dans les premières, les consultations sont brèves, souvent sans rendez-vous, les patients fréquemment inconnus et le suivi n'est pas toujours effectué par le même praticien ; l'interrogatoire, tout comme les conseils de prévention, sont assez standardisés. Les praticiens ont une connaissance relativement superficielle de la situation de leur patient et se montrent désabusés et pessimistes sur l'utilité de leurs conseils de prévention. On peut souligner qu'ils sont plus souvent que les cliniciens libéraux confrontés à des patients ayant des infections sexuellement transmisibles à répétition ou comprenant mal le français. En médecine de ville, les consultations durent plus longtemps, les cliniciens privilégient l'écoute, semblent mener un entretien assez libre et connaissent mieux l'histoire affective et sexuelle de leur patient: sans doute est-ce la raison pour laquelle ils le soupçonnent assez rarement de mentir. À cette opposition public/ privé s'ajoute des différences liées aux représentations qu'ont les médecins de leurs patients. Les médecins qui ont l'habitude de suivre des patients homosexuels considèrent qu'ils n'ont pas à s'immiscer dans leur vie privée et à adopter un discours pouvant paraître moralisateur. Certains refusent de leur recommander d'informer leurs partenaires, estimant que patients et partenaires sont parfaitement informés du mode de transmission et des conséquences de ces infections. À l'opposé, les gynécologues considèrent que les difficultés de prise en charge des partenaires viennent plutôt des partenaires eux-mêmes que de leurs patientes, disposées en général à avertir leurs partenaires actuels et parfois même les anciens. À certains égards, dans leur discours, les femmes apparaissent comme les victimes des hommes, infidèles et irresponsables.

II est important de promouvoir en France, dans les structures recevant des hommes atteints d'infections sexuellement transmissibles ou de $\mathrm{VIH}$, des stratégies de prise en charge du partenaire principal aussi volontaristes que celles des gynécologues. Ces stratégies doivent également concerner l'information des autres partenaires, qui gagnerait à être explicite pour contribuer à limiter la propagation de ces infections dans la population. Mais un programme de santé publique qui se contenterait simplement de rappeler aux cliniciens les bonnes pratiques en la matière a toutes les chances d'être inutile. II faut ainsi convaincre les médecins qu'il est possible et utile d'évoquer de manière systématique et structurée l'information des différents partenaires sexuels de leurs patients, au-delà de la perception qu'ils ont de leurs attentes et de leurs besoins.

\section{Remerciements}

Cette enquête a été financée par l'Agence nationale de recherches sur le sida et les hépatites virales (Anrs). Nous remercions Jean-Pierre Mertz, Philippe Weber, Jean-Claude Desenclos, Anne Laporte, Béatrice Ducot, Sophie Couturier, Emilie Pages, Jocelyn Raude, Laura Beltran et Sylvère Dupont-Monod, ainsi que les médecins et biologistes qui ont participé à cette enquête

\section{Références bibliographiques}

1. Arthur G, Lowndes C, Blackham J, Fenton K, The European surveillance of sexually transmitted infections network. Divergent approaches to partner notification for sexually transmitted infections across the european union. Sexually Transmitted Diseases 2005 ; 32(12) : 734-741.

2. Cowan $F$, French $R$, Johnson $A$. The role and effectiveness of partner notification in STD control : a review. Genitourin Med $1996 ; 72(4): 247-252$.

3. National Health Service. England and Wales (Veneral Diseases). Regulations 1974.

4. Potterat J, Muth S, Muth J. Partner notification early in the aids era: misconstruing contact tracers as bedroom police. Research in Social Policy $1998 ; 6: 1-15$.

5. Wright A, Chippindale S, Mercey D. Investigation into the acceptability and effectiveness of a new contact slip in the management of Chlamydia trachomatis at a London genitourinary medicine clinic. Sex Transm Infect Dec $2002 ; 78(6): 422-424$.

6. Klausner JD, Chaw JK. Patient-delivered therapy for chlamydia: putting research into practice. Sex Transm Dis Jun $2003 ; 30(6)$ : 509-511.

7. Golden $\mathrm{M}$, Whittington $\mathrm{W}$, Handsfield $\mathrm{H}$, et al. Effect of expedited treatment of sex partners on recurrent or persistent gonorrhea or chlamydial infection. New England Journal of Medicine 2005 ; 352 : 676-684.

8. Macke B, Keenan $\mathrm{H}$, Kassler W. Partner notification strategies for sexually transmitted diseases. Sexually Transmitted Diseases 1998 ; july : 329-330.

9. Oxman A, Scott E, Sellors J, et al. Partner notification for sexually transmitted diseases : an overview of the evidence. Can J Public Health 1994 ; Suppl 1: S41-47.

10. Warszawski J. Counseling et notification des partenaires : une analyse coûtefficacité. Transcriptase $2000 ; 80: 2-4$.

11. Faxelid E, Tembo G, Ndulo J, Krantz I. Individual counseling of patients with sexually transmitted diseases. A way to improve partner notification in a Zambian Setting ? Sexually Transmitted Diseases 1996 ; 23 : 289-292.

12. Potterat J, Phillips L, Rothenberg R, Darrow W. Gonococcal pelvic inflammatory disease: case-finding observations. American Journal of Obstetric and Gynecology $1980 ; 138: 1101-1104$.

13. Ross J. How much sexually transmitted disease is not seen by genitourinary physicians ? Genitourin Med $1995 ; 71(5): 333$.

14. Golden MR, Hogben M, Handsfield HH, St Lawrence JS, Potterat JJ, Holmes KK. Partner notification for HIV and STD in the United States : Iow coverage for gonorrhea, chlamydial infection, and HIV. Sex Transm Dis Jun 2003; 30(6) : 490-496.

15. Warszawski J, Meyer L. Apports d'une enquête en population générale à l'épidémiologie des maladies sexuellement transmissibles. In La sexualité au temps du sida, N Bajos, M Bozon, A Ferrand, A Spira, Eds. Paris: PUF, collection Sociologie d'aujourd'hui, 1998, p. 415-435. 
16. Warszawski J, Meyer L. Sex difference in partner notification. Results from three population-based surveys in France. Sexually Transmitted Infections $2002 ; 78: 45-49$.

17. Warszawski J, Bajos N. Sexualité, contraception, MST. In Baromètre santé 2000, P Guilbert, F Baudier, A Gautier, Eds. Vanves: Editions CFES, 2001, p. 209-236.

18. Warszawski J, Meyer L, ACSF Group. Gender difference in persistent at-risk sexual behavior after a diagnosed sexually transmitted disease (STD). Sex Transm Dis $1998 ; 25: 437-442$.
19. Warszawski J, Couturier E, RibertE, et al. Infection sexuellement transmissibles et VIH: les partenaires sexuels sont-ils pris en charge en France? Bulletin Epidémiologique Hebdomadaire 2005 48: 245-246.

20. Georges S, Laurent E, Goulet V, Renachla. Enquête sur les lieux de consultation et les caractéristiques des personnes prélevées pour recherche de Chlamydia trachomatis, 2001. Bulletin Epidémiologique Hebdomadaire 2004; 40-41; 198-199.

21. Herida M. Enquête nationale auprès des Conseils généraux sur les infections à gonocoque diagnostiquées, France métropolitaine, 2001 et 2002. Bulletin Epidémiologique Hebdomadaire $2004 ; 15$ : 60 . 